Oral Presentation

Track 20: Biomechanics of Organs

20.3. Urinary Tract

Abstract: 5565

Citation: Journal of Biomechanics 2006; Vol. 39 Suppl. 1, page S390

\title{
Development of a computational model urethra to study perineal noise as a diagnostic marker for prostatic obstruction
}

\author{
J. Pel, R. van Mastrigt \\ Sector Furore, Department of Urology, Erasmus MC, Rotterdam, Netherlands
}

In men, an enlarging prostate mostly leads to bladder outlet obstruction (BOO). Instead of an invasive pressure/flow study, alternative, non-invasive methods have been proposed to diagnose BOO. One method involves the recording of noise with a contact microphone pressed against the perineum. The noise signal may be caused by turbulence in the urethra, when recirculation of urine is induced by negative pressure values at the end of an obstruction. The streamlines passing the obstruction restore to the urethral wall at the reattachment point. In a number of experimental studies on arterial stenoses, it was shown that the position of maximum wall pressure variation is found just upstream from the reattachment point and that at this location the frequency of passing vortices may be the dominant mechanism of inducing wall vibration [1]. A computational model of a bladder and (rigid) urethra complex was developed to calculate disturbed urinary flow as a result of a narrowed prostatic region using Computational Fluid Dynamics (CFD). The radius of the bladder, the length of the bladder neck, the prostate and the urethra and the diameter of the prostate and the urethra could easily be set to mimic physiological conditions. Calculations were carried out at 4 different bladder pressures and 5 different degrees of obstruction by varying the diameter of the prostatic part. The $\kappa-\varepsilon$ turbulence model was used to calculate flow and pressure distributions along streamlines and wall shear stresses to localise the recirculation zones and the reattachment points. We found that the location of the reattachment point was independent of the applied bladder pressure and depended on the degree of obstruction. Since the reattachment point is possibly the best location to non-invasively measure urethral noise, we aim at extending our CFD urethral model by including urethral visco-elastic properties to develop a non-invasive method for diagnosing BOO based on perineal noise recording.

Reference: $\quad$ 1. J Acoust Soc Am 83: 318. 
